\title{
TIROLORIA
}

\section{Individuazione di un nuovo marker da impiegare per una corretta stadiazione dell'infezione da EBV}

\section{Luana Coltella, Lucia De Florio, Cinzia Manfredini, Fabrizio Bonelli, Daniele Innocenzi, Donato Menichella}

Unità Operativa di Microbiologia, Ospedale Pediatrico Bambino Gesù, Roma

\section{Characterization of a new marker for stage-specific diagnoses of EBV infection}

Key words: EBV, avidity, p I8-specific IgG

\section{SUMMARY}

Epstein Barr Virus (EBV), also classified as Human Herpes Virus 4, infects the vast majority of adults worldwide and establishes both non-productive (latent) and productive (lytic) infection.

Classical EBV diagnosis includes quantitative determination of viral DNA and serological analysis, based on the determination of $\lg G$ and IgM responses against the viral capsid antigen (VCA) and the lgG response against the EBV nuclear antigen-I (EBNA-I). EBV-serology can be misleading in some cases, such as acute infections with low or undetectable VCA IgM, convalescent patients with persistent or reactivated VCA IgM and negative antiEBNA-I IgG, due to a loss of this marker during immunosuppression. In all these cases avidity determination of $\lg \mathrm{g}$ is helpful to prevent false diagnosis.

Avidity represents the stability of the antigen-antibody interaction. Its value increases during the infection, so high avidity never associates with a primary acute infection.

We studied the importance of avidity determination of pl 8 (VCA)-IgG to achieve unequivocal interpretation of serological results.

The amount of $\lg G$ and $\lg M$ is determined by Chemiluminescent Immune Assay (CLIA), a rapid and highly sensitive method suitable for automation. The intensity of luminous signal produced by antibody-antigen recognition is expressed as Relative Light Unit (RLU). p- I8 IgG is determined using a recombinant p/8 antigen expressed in E. coli.

Avidity index is determined in CLIA by the ratio between denaturated and not denaturated IgG specific antibodies expressed in RLU.

These results demonstrate that avidity determination represents an important additional marker particularly in cases with aberrant serological profile.

\section{INTRODUZIONE}

Il virus di Epstein Barr (EBV, HHV4), appartenente alla famiglia dei $\gamma$-Herpesvirus, è un virus linfotropo e ubiquitario con un genoma lineare a DNA a doppio filamento di $172 \mathrm{kbp}$.

La trasmissione del virus avviene principalmente attraverso il contatto con le secrezioni orali come conseguenza della replicazione virale nelle cellule dell'orofaringe.

Il quadro clinico dell'infezione da EBV è estremamente variabile: nell'infanzia è generalmente asintomatica, mentre negli adolescenti e negli adulti si manifesta frequentemente sotto forma di mononucleosi infettiva (MI).

Dopo l'infezione primaria il virus rimane all'interno dei linfociti B della memoria in uno stato di latenza che perdura per tutta la vita dell'individuo, tuttavia, in determinate circostanze, il virus può andare incontro a riattivazione e dare origine ad un'infezione secondaria.
Circa il 90\% della popolazione adulta è sieropositivo per EBV ed è pertanto portatore sano del virus (8).

Nella figura I sono descritte la cinetica di maturazione della risposta anticorpale e l'andamento della carica virale ematica nel corso dell'infezione da EBV.

Durante un'infezione primaria il primo marker anticorpale che compare è costituito dalle IgM dirette verso il complesso VCA (Viral Capsid Antigen). Nel decorso tipico tali anticorpi scompaiono nel giro di 2 o 3 mesi dall'inizio dell'infezione, tuttavia è possibile metterli in evidenza solo nel $70-80 \%$ dei pazienti con infezione primaria acuta (3).

Seguono gli anticorpi IgG anti-EA (Early Antigen), i cui livelli decrescono a valori negativi o molto bassi durante la convalescenza fino a scomparire solitamente nel giro di 6-12 mesi. Queste due frazioni anticorpali possono essere 
messe in evidenza anche nel corso di una riattivazione. Successivamente compaiono gli anticorpi IgG anti-VCA, che persistono per tutta la vita dell'individuo (8). In particolare tali anticorpi sono costituiti da più sottopopolazioni, tra cui le $\operatorname{IgG}$ dirette contro la proteina p23 (10), che solitamente compaiono subito dopo le IgM anti-VCA, e le IgG dirette contro la proteina p18 (14), che seguono le precedenti e rappresentano pertanto un maker più tardivo. Solo dopo settimane o mesi dall'inizio dell'infezione vengono tipicamente evidenziati gli anticorpi IgG anti-EBNA-1 (EBV Nuclear Antigen), la cui presenza costituisce pertanto un criterio per escludere un'infezione primaria. Circa il 5\% della popolazione infettata da EBV non produce anticorpi IgG anti-EBNA-1 anche a distanza di mesi, simulando quindi un'infezione primaria (3); inoltre tali anticorpi possono scomparire in seguito a sopravvenuta immunosoppressione (3). La loro assenza può essere pertanto indice sia di un'infezione recente sia di una mancata produzione o perdita successiva di questa frazione anticorpale.

Attualmente la diagnosi di infezione da EBV si esegue con la ricerca del genoma virale mediante PCR Real Time e con la ricerca degli anticorpi specifici di classe $\operatorname{IgM}$ e IgG diretti contro gli antigeni virali capsidici (VCA) e degli anticorpi IgG diretti contro l'antigene nucleare EBNA1.Tuttavia, a causa del comportamento non canonico dei marker anticorpali, la sierologia classica non sempre permette una diagnosi inequivocabile della fase dell'infezione. Questo accade ad esempio nel caso di infezione acuta in presenza di livelli bassi o non rilevabili di IgM anti-VCA, o nella fase di convalescenza con IgM anti-VCA persistenti e IgG anti-EBNA-1 assenti $(2,9)$.

In quelle infezioni, virali e non, in cui è fondamentale determinare lo stadio dell'infezione e/o distinguere tra infezioni primarie e riattivazioni, si è rivelata particolarmente utile la determinazione dell'avidità anticorpale.

Il termine "avidità" viene usato comunemente per descrivere il grado di resistenza, da parte del complesso antigene-anticorpo, nei confronti della dissociazione operata da agenti denaturanti (dietilammina, urea $8 \mathrm{M}$ ). Durante la maturazione della risposta umorale l'affinità degli anticorpi di classe IgG diretti verso i vari antigeni aumenta nel tempo, determinando pertanto una maggiore stabilità del complesso antigene-anticorpo e quindi un maggiore Indice di Avidità (IA) anticorpale.

\section{Scopo del Lavoro}

Lo scopo del lavoro è stato quello di valutare l'impiego dell'avidità delle IgG anti-EBV come mezzo diagnostico per operare una corretta sta- diazione dell'infezione nei casi in cui gli altri marker sierologici non siano sufficienti a tal fine. In particolare è stato valutato l'impiego di una metodica in chemiluminescenza per determinare l'indice di avidità degli anticorpi di classe IgG diretti verso l'antigene p18 (VCA). Dal momento che questi anticorpi vengono prodotti tardivamente, la determinazione del loro indice di avidità può essere considerata particolarmente utile nei casi in cui gli anticorpi di classe IgG anti EBNA-1 siano assenti.

\section{MATERIALI E METODI}

\section{Scelta della popolazione}

Lo studio è stato condotto su un totale di 252 sieri relativi a 230 soggetti "immunocompetenti" (soggetti non immunocompromessi congenitamente o per infezione diversa da EBV, né sottoposti a terapia immunosoppressiva) conservati a $-20^{\circ} \mathrm{C}$ nella sieroteca del Laboratorio di Microbiologia dell'Ospedale Pediatrico Bambino Gesù di Roma. Su questi sieri sono state eseguite le analisi sierologiche in Immunoblot, "gold standard" di riferimento nella diagnosi di infezione da EBV (3), e in chemiluminescenza, ed è stata eseguita la determinazione della carica virale nel sangue intero e nel plasma mediante PCR Real Time.

Sulla base dei risultati ottenuti con l'Immunoblot, prendendo come marker di riferimento gli anticorpi di classe IgM in toto, le IgG dirette contro le due frazioni del VCA e le IgG anti-EBNA-1, i 252 sieri sono stati classificati in 5 profili anticorpali (tabella 1).

\section{Immunoblot}

Il test in Immunoblot è stato eseguito con l'impiego del kit recomLine EBV IgG e IgM (MIKROGEN, Germania). Il campione di siero viene diluito e incubato con strip di nitrocellulosa su cui sono "blottati" gli antigeni ricombinanti di EBV: EBNA-1, le componenti p18 e p23 del VCA e le due componenti degli EA (p138, p54). Dopo lavaggio le strip vengono ulteriormente incubate con anticorpi anti $\operatorname{IgG}$ o IgM umane, coniugate con l'enzima perossidasi di rafano. Gli anticorpi eventualmente presenti nel campione si legano in modo specifico alla strip e vengono messi in evidenza da una reazione colorimetrica, catalizzata dalla perossidasi, sotto forma di una banda scura in corrispondenza del locus antigenico specifico.

\section{Saggio Immunometrico in Chemiluminescenza} (CLIA)

Gli anticorpi specifici (IgG e IgM) diretti verso i vari antigeni di EBV sono stati determinati quantitativamente impiegando un test indiretto basato sul principio della chemiluminescenza (CLIA). La determinazione è stata effettuata mediante il sistema automatico LIAISON DiaSorin con l'impiego dei seguenti kit: LIAISON EBV IgM, 
LIAISON VCA IgG, LIAISON EBNA IgG (DiaSorin SpA, Italia).

Gli antigeni specifici sono adesi a particelle magnetiche (fase solida) e durante la prima incubazione vengono legati dagli anticorpi specifici presenti nel campione di siero.

Nella seconda incubazione un anticorpo monoclonale di topo anti-anticorpi umani, coniugato con un derivato dell'isoluminolo, reagisce con gli anticorpi legati alla fase solida; in seguito all'aggiunta dei reagenti starter viene indotta la reazione di chemiluminescenza. La quantità di anticorpi presenti nel campione è proporzionale all'intensità del segnale luminoso prodotto, misurato in unità relative di luce (RLU).

Per la ricerca degli anticorpi IgG diretti verso la proteina $\mathrm{p} 18$ sono stati utilizzati antigeni ricombinanti (regione corrispondente agli aminoacidi 322-534) espressi in E. coli.

\section{Determinazione in CLIA dell'indice di Avidità delle IgG anti-p18}

Per la determinazione dell'indice di avidità è stato impiegato un kit prototipo LIAISON VCA-G Avidity fornitoci dalla DiaSorin SpA.

L'indice di avidità delle IgG valuta la forza del legame antigene-anticorpo. Viene determinato dal rapporto tra le RLU degli aticorpi IgG specifici trattati con l'agente denaturante (Urea $8 \mathrm{M}$ ) e le RLU degli anticorpi IgG specifici non trattati.

\section{Determinazione della carica virale}

Il DNA virale viene estratto dal sangue intero e dalla componente plasmatica, ottenuta dopo centrifugazione del campione, con l'impiego del kit QIAamp DNA Mini Kit (QIAGEN, Germania) secondo le indicazioni fornite dalla ditta produttrice. La determinazione quantitativa del genoma virale viene eseguita mediante PCR Real Time (ABI Prism 7900 Sequence Detector, Applied Biosystems, metodica TaqMan), usando come target la regione Bam HI-W di EBV. Il numero di copie genomiche per $\mathrm{ml}$ di sangue viene calcolato sulla base di una curva standard costruita amplificando tre diverse concentrazioni dello standard fornito, costituito dal DNA target clonato in un plasmide (EBV DNA Regione Bam HIW, Alfa Wassermann).

\section{RISULTATI}

Determinazione della Soglia dell'Indice di Avidità delle IgG anti-p18 nei Soggetti Immunocompetenti

Lo studio è volto a definire un cut-off, per l'indice di avidità delle IgG anti-p18, al di sotto del quale considerare un'infezione primaria in fase acuta anche in assenza del marker di infezione precoce IgM anti-VCA e al di sopra del quale escluderla, anche in assenza del marker di latenza
EBNA-1 IgG e/o in presenza di IgM anti-VCA. Lo studio è stato inizialmente condotto sui sieri con pattern anticorpale riferibile ad un'infezione pregressa: assenza degli anticorpi IgM anti-VCA, presenza degli anticorpi IgG anti-VCA e presenza degli anticorpi IgG anti-EBNA-1 (profilo “- ++"). Il profilo sierlogico "- + +" è caratteristico dei soggetti che hanno risolto l'infezione da più o meno tempo: nel 95\% dei sieri entrambi i marker di replicazione virale (IgG anti-EA e genoma virale) sono assenti o presenti a basso titolo (tabella 2).

Quei sieri che presentano ancora segni di replicazione virale sono riferibili a diverse situazioni cliniche: transizione dell'infezione dalla fase acuta alla fase di convalescenza, superamento dell'infezione con persistenza di bassi livelli del/i suddetti marker, riattivazione dell'infezione.

Per i 57 sieri appartenenti a questo profilo sierologico è stato misurato l'Indice di Avidità (AI) delle IgG anti-p18. La distribuzione dei valori ottenuti è mostrata in figura II.

Tale distribuzione evidenzia che tutti i sieri presentano un Indice di Avidità delle IgG anti p-18 superiore al valore di $0.300 \mathrm{AI}$, indicando quest'ultimo come il possibile cut-off al di sotto del quale un'infezione può essere definita primaria.

Gli 8 sieri che hanno Indice di Avidità compreso fra 0.300 e 0.400 AI presentano ancora segni di replicazione virale (positività alla PCR e/o presenza del marker IgG anti-EA), suggerendo una condizione di transizione o di recente risoluzione dell'infezione. Dei restanti 49 sieri, tutti con Indice di Avidità $>0.400$, il $71 \%$ (35 sieri) non mostra né il marker IgG anti-EA né la presenza del genoma virale, confermando una situazione di infezione pregressa. Il $25 \%$ (12 sieri) mostra bassi livelli di positività solo per uno dei due marker di replicazione virale, suggerendo una risoluzione dell'infezione con persistenza del marker a livelli prossimi al limite di sensibilità. Il restante $4 \%$ (2 sieri) presenta invece alta carica virale e alti livelli di IgG anti-EA, indicando presumibilmente una riattivazione dell'infezione.

La conferma del valore soglia precedentemente ipotizzato è stata effettuata attraverso l'analisi dei sieri con pattern anticorpale solitamente riferibile ad un'infezione primaria in fase acuta (12): presenza dei marker IgG e IgM anti-VCA e assenza degli anticorpi anti-EBNA-1 (profilo “+ + -"). Trattandosi di una situazione opposta alla precedente, infatti, la distribuzione dei sieri appartenenti a questo profilo dovrebbe prevalentemente disporsi al di sotto del cut-off precedentemente definito.

In base ai risultati dell'Immunoblot, gli 85 sieri relativi a questo quadro sierologico si presentano suddivisi in due sottopopolazioni: 37 sieri presentano esclusivamente gli anticorpi IgG anti-p23 e 48 sieri presentano gli anticorpi IgG diretti verso 
entrambe le componenti del VCA (tabella 3).

Tuttavia, dal momento che in chemiluminescenza tutti i sieri considerati sono risultati positivi per le IgG anti-p18, lo studio è stato esteso anche ai 37 sieri risultati all'Immunoblot positivi solo per le IgG anti-p23.

Il 93\% dei sieri appartenenti a questo profilo presenta segni di replicazione virale (PCR positiva e/o IgG anti-EA), inoltre, nella maggior parte dei casi, i due marker sono presenti contemporaneamente a confermare lo stato di infezione acuta (tabella 4).

La valutazione dell'Indice di Avidità delle $\operatorname{IgG}$ anti-p18 dei sieri relativi al profilo " ++ + " ha mostrato la distribuzione dei valori riportata nella figura III.

Anche in questo caso il valore soglia precedentemente identificato risulta indicativo, dal momento che $1^{\prime} 85 \%$ ( 72 sieri) si distribuisce al di sotto del valore di $0.300 \mathrm{AI}$, evidenza di una recente comparsa della frazione anticorpale IgG anti-p18 e quindi di un'infezione primaria in fase acuta. Ciò è confermato anche dal fatto che i 72 sieri presentano tutti almeno uno dei due marker di replicazione virale. L'8\% (7 sieri) rientra nell'intervallo compreso tra 0.300 e $0.400 \mathrm{AI}$, indice di una situazione di evoluzione verso una risoluzione dell'infezione. Solo il 7\% (6 sieri) presenta valori superiori a $0.400 \mathrm{AI}$, indice di una situazione diversa da un'infezione primaria e imputabile ad altre situazioni cliniche: riattivazione dell'infezione, infezione cronica attiva, risoluzione dell'infezione con persistenza del marker IgM anti-VCA e contemporanea assenza o ritardo nella comparsa del marker IgG anti-EBNA-1. In questi casi la valutazione di altri parametri è di ausilio per meglio definire il quadro clinico.

Ad esempio, di questi 6 sieri, 2 presentano alti valori dei marker di replicazione virale, suggerendo l'ipotesi o di una riattivazione dell'infezione o di un'infezione cronica attiva (CAEBV). I restanti 4 , che non presentano segni di replicazione, si riferiscono ad una risoluzione dell'infezione con un ritardo o una mancata comparsa degli anticorpi IgG anti-EBNA-1.

La definizione del valore soglia per l'avidità delle IgG anti-p18 risulta evidente dalla sovrapposizione delle distribuzioni relative ai due profili analizzati (figura IV).

Si osserva infatti la presenza di un cut-off discriminante tra le due distribuzioni in corrispondenza del valore di $0.300 \mathrm{AI}$, al di sotto del quale un'infezione può sicuramente essere considerata acuta, e al di sopra della quale non può essere considerata primaria. Vi è inoltre una stretta fascia di sovrapposizione tra le due distribuzioni, in cui i sieri hanno un valore di avidità intermedio com- preso tra $0.300-0.400 \mathrm{AI}$. In questo intervallo sono presenti un numero approssimativamente uguale di sieri relativi a ciascuno dei due profili sierologici e rappresenta pertanto una situazione di transizione tra due fasi successive dell'infezione.

\section{Studio della Cinetica di Maturazione dell'Avidità delle IgG anti-p18}

Per completare il quadro della cinetica di maturazione dell'avidità delle IgG anti-p18, lo stesso studio è stato esteso ai sieri relativi agli altri due profili sierologici considerati: quello che presenta esclusivamente gli anticorpi IgG anti-VCA (“- + -") e quello che presenta contemporaneamente i tre marker anticorpali ("+++"). Rispetto ai quadri sierologici precedentemente considerati, la distribuzione dei sieri relativi a questi due profili si è rivelata più eterogenea (figura V).

La ragione è da attribuire al comportamento non canonico dei marker sierologici, per cui uno stesso profilo anticorpale può essere riconducibile a diverse situazioni cliniche (infezione primaria, situazione di transizione, risoluzione dell'infezione, riattivazione), ciascuna caratterizzata però da un diverso valore di avidità delle IgG anti-p18. Nel profilo "- + _" i sieri con avidità inferiore a $0.300 \mathrm{AI}$ rappresentano un'infezione primaria in cui gli anticorpi IgM o non vengono prodotti o sono al di sotto del limite di sensibilità del test.

I sieri con avidità compresa tra $0.300-0.400 \mathrm{AI}$ si riferiscono a situazioni di transizione verso la risoluzione dell'infezione; quelli con indice di avidità superiore a 0.400 AI si riferiscono a risoluzioni senza produzione o con perdita degli anticorpi IgG anti-EBNA-1, o a rare situazioni di riattivazione dell'infezione senza produzione di IgM e in assenza di IgG anti-EBNA-1.

La distribuzione dei sieri verso valori di avidità alti è indice della prevalenza, in questo profilo, di situazioni di risoluzione dell'infezione in soggetti che non producono o tardano a produrre IgG anti-EBNA-1. Nel profilo " +++ " i sieri con avidità inferiore a $0.300 \mathrm{AI}$ sono rari e possono essere riferibili ad un'infezione acuta con precoce comparsa del marker IgG anti-EBNA-1. Anche in questo profilo i sieri si distribuiscono principalmente verso valori di media e soprattutto alta avidità $(>0.400$ $\mathrm{AI})$, riferibili a situazioni di transizione/risoluzione dell'infezione con persistenza di IgM o a situazioni di riattivazione.

La presenza nel 55\% dei sieri di segni di replicazione virale e/o del marker IgG anti-EA, conferma che proprio in questo profilo rientrano la maggior parte delle situazioni di riattivazione (dati non mostrati).

Nella figura VI è riportata la distribuzione dei sieri relativi ai quattro profili sierologici in relazione al valore di avidità delle IgG anti-p18. 


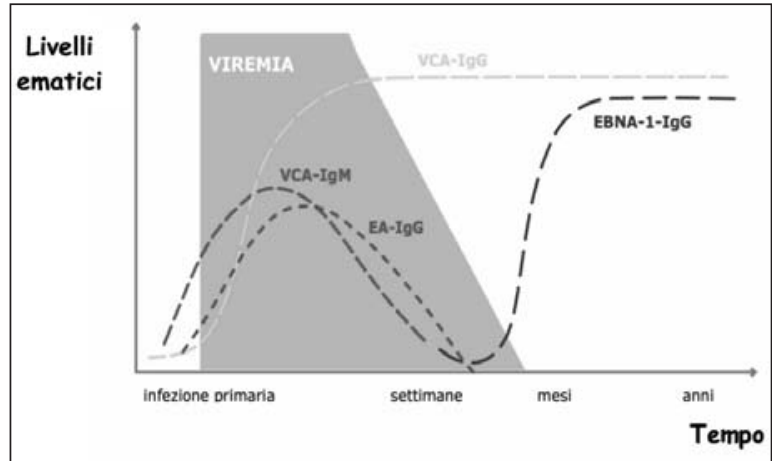

Figura I. Cinetica di maturazione della risposta anticorpale (curve tratteggiate) e andamento della carica virale ematica nel corso dell'infezione da EBV (ombreggiatura grigia).

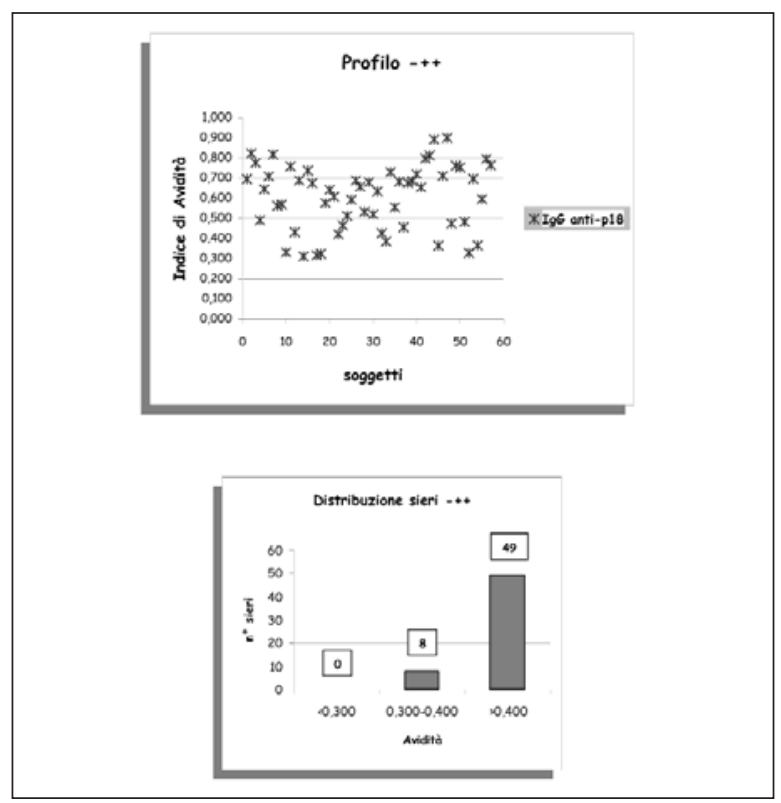

Figura II. Distribuzione dei sieri con profilo “- + +" in relazione al valore dell'Indice di Avidità delle lgG anti-p/8

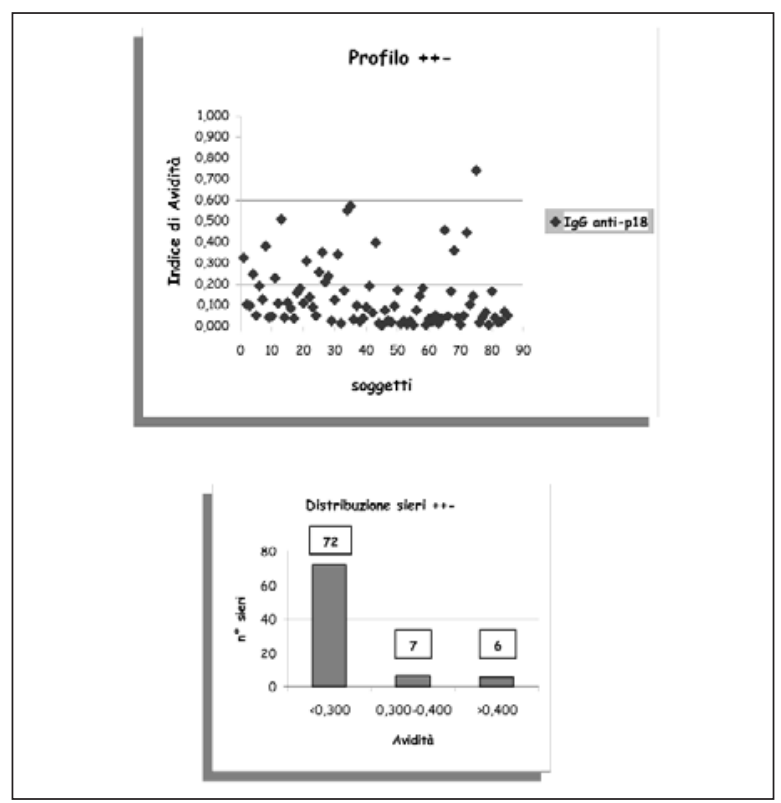

Figura III. Distribuzione dei sieri con "profilo + + -" in relazione al valore dell'Indice di Avidità delle lgG anti-p/ 8

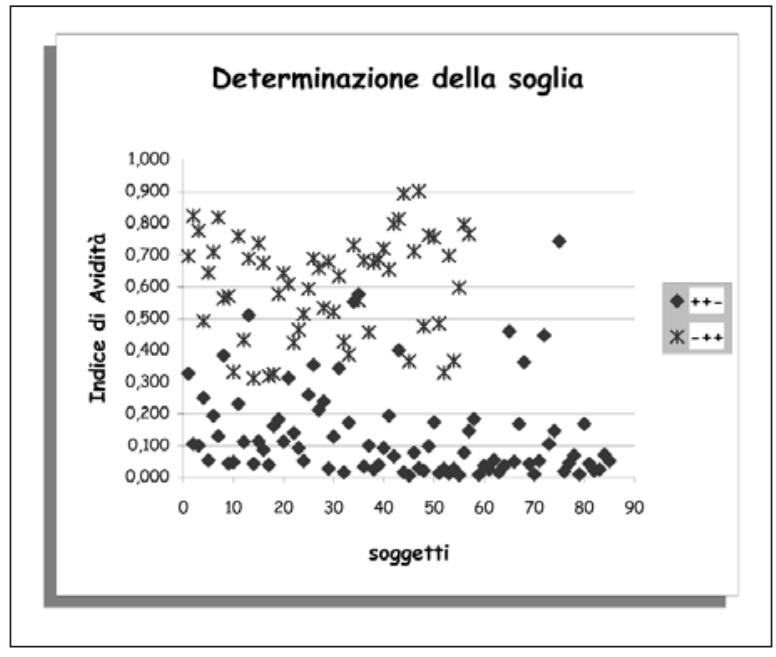

Figura IV. Determinazione della soglia di avidità anticorpale attraverso la sovrapposizione delle distribuzioni dei sieri con profilo “- + +" e“+ + -"

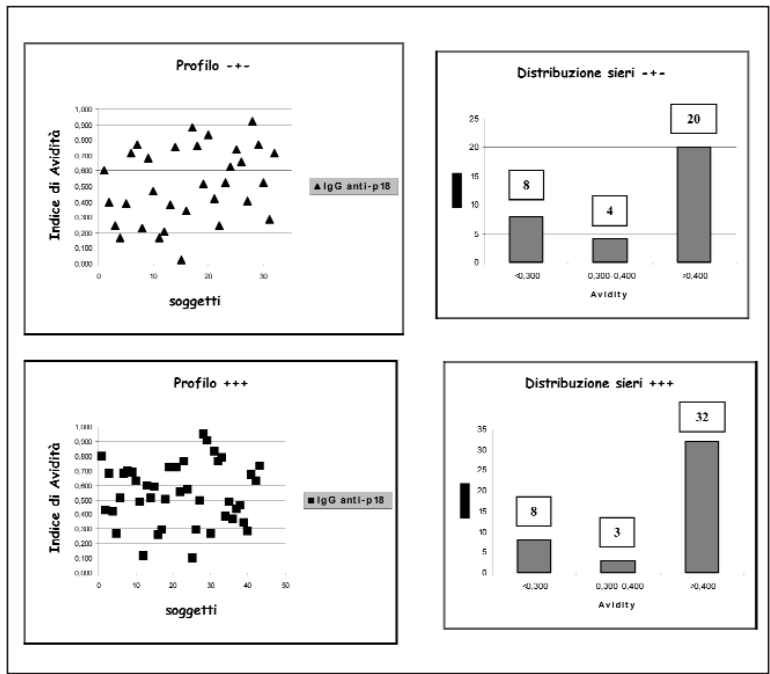

Figura V. Distribuzioni dei sieri con profilo “- + -” " “+ + +" in relazione al valore dell'Indice di Avidità delle lgG anti-p/8

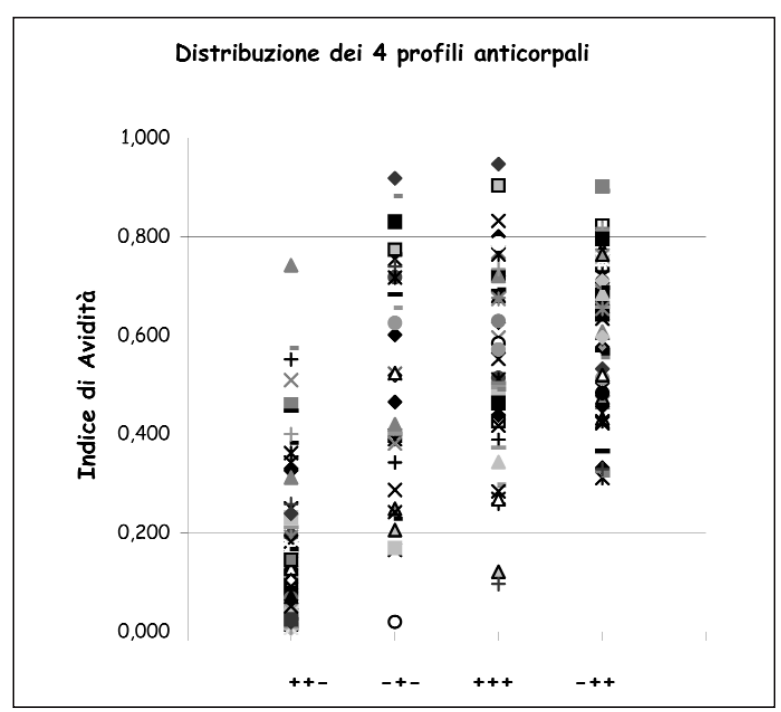

Figura VI. Distribuzione dei sieri relativi ai 4 profili anticorpali in relazione al valore dell'Indice di Avidità delle lgG anti-p 18 


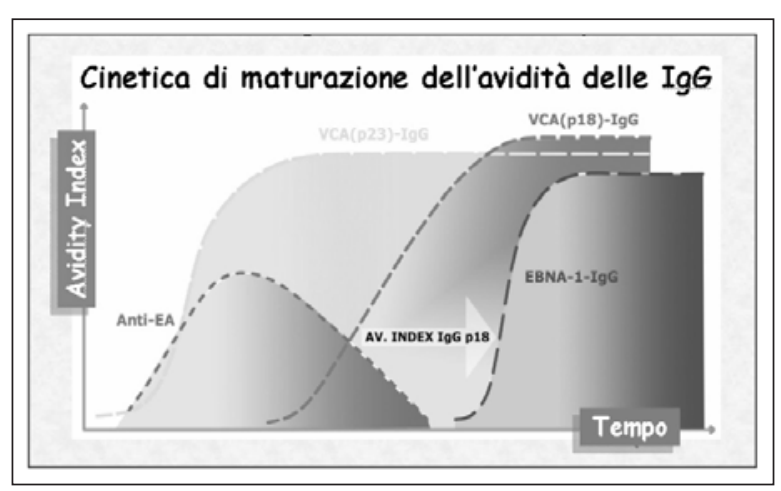

Figura VII. Cinetica di maturazione dell'indice di avidità degli anticorpi IgG diretti contro le diverse frazioni antigeniche di EBV

Tabella I. Distribuzione dei 252 sieri nei 5 profili anticorpali risultanti dall'analisi mediante Immunoblot

\begin{tabular}{|c|c|c|c|}
\hline $\begin{array}{l}V C A \\
\text { IgM }\end{array}$ & $\begin{array}{l}\mathrm{VCA} \\
\mathrm{Ig} G\end{array}$ & $\underset{I g G}{E B N A-1}$ & $\begin{array}{c}\text { Soggetti } \\
\text { "Immunocompetentir" }\end{array}$ \\
\hline - & - & - & 35 \\
\hline+ & + & - & 85 \\
\hline+ & + & + & 43 \\
\hline- & + & - & 32 \\
\hline- & + & + & 57 \\
\hline \multicolumn{3}{|c|}{ Totale } & 252 \\
\hline
\end{tabular}

Tabella 2. Distribuzione dei 57 sieri relativi al profilo “- + +" in funzione della presenzalassenza delle lgG anti-EA e del genoma di EBV.



Tabella 3. Distribuzione degli 85 sieri relativi al "profilo + + _" risultante dall'analisi mediante Immunoblot

\begin{tabular}{|c|c|c|c|c|}
\hline $\begin{array}{l}\text { VCA } \\
\text { IgM }\end{array}$ & $\begin{array}{l}\mathrm{p} 23 \\
\mathrm{IgG}^{2}\end{array}$ & $\begin{array}{l}\mathrm{P} 18 \\
\mathrm{Ig} G\end{array}$ & $\underset{I g G}{\text { EBNA-1 }}$ & $\begin{array}{c}\text { Soggetti } \\
\text { "Immunocompetenti" }\end{array}$ \\
\hline+ & + & - & - & 37 \\
\hline+ & + & + & - & 48 \\
\hline \multicolumn{3}{|c|}{ Totale } & & 85 \\
\hline
\end{tabular}

\section{Confronto della Metodica in Chemiluminescenza con il Gold Standard di Riferimento: l'Immunoblot}

Nel corso della valutazione della presenza dei marker sierologici nei campioni relativi al profilo "+ + _" sono emerse alcune discordanze tra i risultati ottenuti utilizzando l'Immunoblot e quelli
Tabella 4. Distribuzione degli 85 sieri relativi al "profilo + + _" in funzione della presenzalassenza delle IgG anti-EA e del genoma di EBV

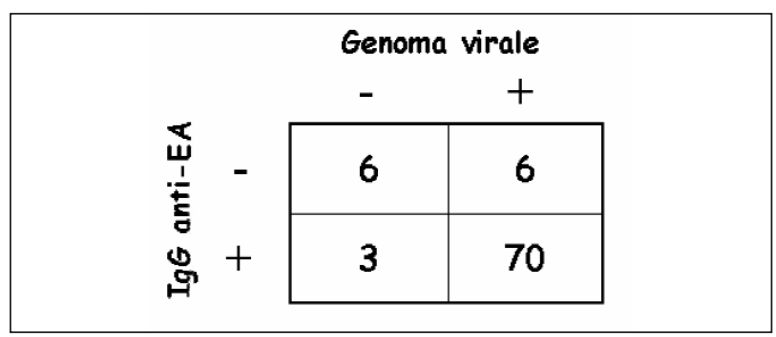

ottenuti con l'analisi in chemiluminescenza, in particolare nella valutazione della presenza delle IgG anti-p18 (tabella 3). Alla luce delle valutazioni fatte in merito all'avidità, si osserva che più del $90 \%$ dei sieri in cui l'Immunoblot non aveva evidenziato presenza di IgG anti-p18 sono associati ad un valore di avidità inferiore a $0.300 \mathrm{AI}$ (dati non mostrati). Ciò suggerisce che, nella rilevazione di questo parametro, la minore sensibilità dell'Immunoblot rispetto al saggio in chemiluminescenza sia imputabile ad una maggiore dipendenza di questa metodica dalla forza del legame antigene-anticorpo.

In presenza di anticorpi caratterizzati da una bassa avidità, quindi, i numerosi lavaggi e l'agitazione meccanica possono determinare un distacco dell'anticorpo dal filtro di ibridazione, che si traduce in un'assenza di segnale.

\section{DISCUSSIONE}

Per la diagnosi di infezione da EBV sono attualmente disponibili ed utilizzati di routine metodi di indagine diretti e indiretti (7); mentre il monitoraggio dell'infezione nei pazienti immunocompromessi si esegue preferibilmente con l'impiego di metodi diretti, come la determinazione della carica virale $(5,6)$.

Sebbene un'infezione primaria da EBV spesso progredisce in modo asintomatico e i casi clinici di mononucleosi non rappresentano un problema, è spesso richiesta la diagnosi differenziale tra un'infezione acuta da EBV e infezioni acute da CMV, HIV, HAV, HBV, HCV, virus della rosolia e del morbillo. È inoltre utile la diagnosi differenziale con infezioni da virus neurotropici, toxoplasmosi, brucellosi, leptospirosi, infezione streptococcica, difteria, linfoma e leucemia (2).

L'indagine sierologia risulta particolarmente utile per la diagnosi differenziale e per determinare la fase dell'infezione, operando una distinzione accurata fra infezione primaria, risoluzione e riattivazione dell'infezione (3). Tuttavia, la grande variabilità di comportamento dei marker comunemente considerati (IgM anti-VCA, IgG antiVCA, IgG anti-EBNA-1) spesso non consente di determinare in maniera univoca lo stadio dell'in- 
fezione $(2,4)$. L'introduzione della valutazione dell'Indice di Avidità (AI) degli anticorpi di classe IgG diretti verso la componente virale capsidica (VCA) si è rivelato un parametro particolarmente significativo nella diagnostica dell'infezione da $\operatorname{EBV}(1,11)$.

Nell'ambito di questo studio è stato valutato l'impiego di una metodica in chemiluminescenza per la determinazione dell'indice di avidità degli anticorpi $\operatorname{IgG}$ diretti verso la componente $\mathrm{p}-18$ del VCA. Dal momento che le IgG anti-p18 vengono prodotte tardivamente, la determinazione del loro indice di avidità può essere considerata particolarmente utile nei casi in cui siano assenti gli anticorpi IgG anti EBNA-1, la cui presenza costituisce un criterio per escludere un'infezione primaria. In particolare, la determinazione dell'avidità delle IgG anti-p18, a differenza dell'avidità delle IgG anti-VCA in toto, non risente della precoce comparsa e quindi maturazione, degli anticorpi IgG anti-p23, come mostrato nella figura VII, che riporta la cinetica di maturazione dell'avidità delle IgG di tutte le frazioni anticorpali prodotte durante l'infezione da EBV.

Dallo studio condotto su 252 sieri relativi a 230 soggetti immunocompetenti sieropositivi per EBV è emerso che un valore superiore a $0.300 \mathrm{AI}$ permette sempre di escludere un'infezione primaria in fase acuta, in presenza o assenza degli anticorpi IgG anti-EBNA-1, e nella maggior parte dei casi definisce il superamento dell'infezione.

L'avidità delle IgG anti-p18 permette di discriminare diverse situazioni cliniche che all'analisi dei marker classici sono caratterizzate da uno stesso quadro sierologico. In particolare permette di differenziare un'infezione primaria da un'infezione pregressa in soggetti che non presentano anticorpi IgG anti-EBNA1, situazione particolarmente frequente nei pazienti immunocompromessi (2). Permette inoltre di discriminare, in assenza del marker IgG anti-EBNA1, tra quei casi in cui gli anticorpi IgM anti-VCA sono dovuti ad un'infezione primaria in fase acuta e quelli in cui sono conseguenza di una riattivazione dell'infezione (frequente nei soggetti immunocompromessi) o di una non risoluzione (13), come nel caso dell'infezione cronica attiva (CAEBV).

L'avidità delle IgG anti-p18 risulta pertanto particolarmente utile per prevenire false diagnosi soprattutto nel caso dell'ospite immunocompromesso, in cui è frequente il quadro sierologico caratterizzato da presenza di anticorpi VCA-IgM e assenza degli anticorpi IgG anti-EBNA1. In questi casi solo il valore dell'avidità permette di discriminare tra un'infezione primaria in fase acuta e una riattivazione. Una riattivazione dell'infezione rappresenta la ripresa della fase litica in un soggetto che presenta il virus in forma latente. L'entità di tale ripresa può essere molto piccola e di breve durata, come nel caso di disturbi a breve termine del sistema immunitario, causati ad esempio da altre infezioni. In altri casi può essere invece di lunga durata e massiva, come in pazienti con difetti immunologici o sottoposti a terapia immunosoppressiva. In questi casi esiste il pericolo di sviluppare un linfoma EBV-associato, come nel caso di pazienti sottoposti a terapia immunosoppressiva in seguito a trapianto d'organo o di midollo. Nei pazienti pediatrici il rischio di sviluppare una malattia linfoproliferativa post trapianto (PTLD) o un altro disordine EBV-correlato è circa 5 volte maggiore dell'adulto. La ragione sta nel fatto che un'infezione primaria progredisce più rapidamente verso una malattia linfoproliferativa rispetto ad una riattivazione secondaria, ed è più probabile che un soggetto pediatrico, rispetto ad un soggetto adulto, sia sieronegativo al momento del trapianto.

L'ampliamento della diagnosi sierologica con l'introduzione dell'indice di avidità delle $\mathrm{IgG}$ anti p-18 costituisce un'implementazione diagnostica di importante ricaduta clinica.

I metodi di indagine sierologica, poco invasivi dal punto di vista del paziente, risultano particolarmente vantaggiosi sia sul piano della rapidità $\mathrm{e}$ facilità di esecuzione, sia sul piano economico, poiché una maggiore consapevolezza dello stadio dell'infezione è finalizzata al risparmio di terapie incongrue nel paziente.

\section{BIBLIOGRAFIA}

1. Andersson A, Vetter V, Kreutzer L, Bauer G. Avidities of $\mathrm{IgG}$ directed against viral capsid antigen or early antigen: useful markers for significant Epstein-Barr virus serology. J Med Virol 1994; 43(3): 238-44.

2. Bauer $\mathrm{G}$. The rational basis for efficient Epstein-Barr virus (EBV) serology. Clin Lab 1995; 41: 623-34.

3. Bauer G. Simplicity Through Complexity: Immunoblot With Recombinant Antigens as the New Gold Standard in Epstein-Barr Virus Serology. Clin Lab 2001; 47: 223-30.

4. Gartner BC, Kortmann K, Schaefer M, MuellerLantzsch N. No correlation in Epstein-Barr virus reactivation between serological parameters and viral load. J Clin Microbiol 2000; 38(6): 2458

5. Gartner BC, Schafer H, Marggraff K, et al. Evaluation of use of Epstein-Barr viral load in patients after allogeneic stem cell transplantation to diagnose and monitor posttransplant lymphoproliferative disease. J Clin Microbiol 2002; 40(2): 351-8.

6. Gartner BC, Fischinger J, Schafer H, Einsele H, Roemer K, Muller-Lantzsch N. Epstein-Barr viral load as a tool to diagnose and monitor post-transplant lymphoproliferative disease. Recent Results Cancer Res 2002; 159: 49-54.

7. Hess RD. Routine Epstein-Barr Virus diagnostics from the Laboratory Perspective: Still Challenging 
after 35 Years. J Clin Microbiol 2004; 42(8): 3381-7.

8. Lennette ET. Epstein-Barr virus. In PR Murray, EJ Baron, MA Pfaller, FC Tenover, and RH Yolken (ed.), Manual of clinical microbiology, 6th ed. ASM Press, Washington DC 1995; 905-10.

9. Rea TD, Ashley RL, Russo JE, Buchwald DS. A systematic study of Epstein-Barr virus serologic assays following acute infection. Am J Clin Pathol 2002; 117(1): 156-61.

10. Reischl U, Gerdes C, Motz M, Wolf H. Expression and purification of an Epstein-Barr virus encoded 23$\mathrm{kDa}$ protein and characterization of its immunological properties. J Virol Methods 1996; 57(1): 71-85.

11. Robertson P, Beynon S, Whybin R, Brennan C, Vollmer-Conna U, Hickie I, Lloyd A. Measurement of EBV-IgG anti-VCA avidity aids the early and reliable diagnosis of primary EBV infection. J Med Virol 2003; 70(4): 617-23.

12. Shillinger M, Kampmann M, Henninger K, Murray G, Hanselmann I, Bauer G. Variability of humoral immune response to acute Epstein Barr Virus (EBV) infection: evaluation of the significance of serological markers. Medical Microbiology Letters 2: 296-303.

13. Schubert J, Zens W, Weissbrich B Comparative evaluation of the use of immunoblots and of IgG avidity assays as confirmatory tests for the diagnosis of acute EBV infections. J Clin Virol 1998; 11(3): 161-72.

14. Van Grunsven WM, van Heerde EC, de Haard HJ, Spaan WJ, Middeldorp JM. Gene mapping and expression of two immunodominant Epstein-Barr virus capsid proteins. J Virol 1993; 67(7): 3908-16.

\section{Luana Coltella}

Ospedale Pediatrico Bambino Gesù

Piazza S. Onofrio 4, 00165 Roma

Tel. 06 68592599; Fax 0668592218

E-mail: coltella@hotmail.com 